\section{Enhancer-promoter communication mediated by Chip during Pannier-driven proneural patterning is regulated by Osa}

\author{
Pascal Heitzler, ${ }^{1}$ Luc Vanolst, Inna Biryukova, \\ and Philippe Ramain ${ }^{2}$ \\ Institut de Génétique et de Biologie Moléculaire et Cellulaire, \\ CNRS/INSERM/ULP, 67404 Illkirch Cedex, \\ Strasbourg, France
}

The GATA factor Pannier activates proneural achaete/ scute $(a c / s c)$ expression during development of the sensory organs of Drosophila through enhancer binding. Chip bridges Pannier with the (Ac/Sc)-Daughterless heterodimers bound to the promoter and facilitates the enhancer-promoter communication required for proneural development. We show here that this communication is regulated by Osa, which is recruited by Pannier and Chip. Osa belongs to Brahma chromatin remodeling complexes and we show that Osa negatively regulates $a c / s c$. Consequently, Pannier and Chip also play an essential role during repression of proneural gene expression. Our study suggests that altering chromatin structure is essential for regulation of enhancer-promoter communication.

Received November 26, 2002; revised version accepted January 8, 2003.

Transcription factors must gain access to their target sites in vivo to regulate gene expression and this implies alteration of chromatin structure around regulatory regions by chromatin remodeling complexes. In Drosophila, the polycomb group of genes maintains repression of homeotic genes like Ultrabithorax, presumably by inducing a repressive chromatin structure (Pirotta 1997). In contrast, some members of the trithorax group of genes were identified by their ability to suppress dominant polycomb phenotypes (Kennison and Tamkun 1998). The os a trithorax group gene encodes a large ubiquitously expressed protein with an ARID domain (․ㅡ/TRich Interaction Domain; Treisman et al. 1997; Collins et al. 1999/ that binds AT-rich DNA sequences, and a C-terminal domain, the eyelid homologous domain (EHD) whose function is not known. The EHD is conserved in multicellular organisms including Caenorhabditis elegans and humans but is not present in yeast. Osa is a component of Brahma (Brm) chromatin remodeling

[Keywords: Transcription; enhancer-promoter communication; chromatin; Osa]

Corresponding authors.

${ }^{1}$ E-MAIL pascal@igbmc.u-strasbg.fr; FAX 33-03-88-65-33-01.

${ }^{2}$ E-MAIL phr@igbmc.u-strasbg.fr; FAX 33-03-88-65-33-01.

Article and publication are at http://www.genesdev.org/cgi/doi/10.1101/ $\operatorname{gad} .255703$. complexes (Treisman et al. 1997; Collins et al. 1999), the Drosophila homologs of the yeast SWI/SNF. These Brm complexes are believed to play a crucial role during gene expression by regulating chromatin structure. However, how these complexes are recruited to specific genes remains poorly understood.

The patterning of the large sensory bristles (macrochaetae) of the thorax of Drosophila is a classical model to study how a specific pattern is generated during development. There are only 11 sensory organs on each heminotum and they occupy stereotyped positions. The development of the bristle precursor depends on expression of the achaete/scute $(a c / s c)$ proneural genes (Modolell 1997). Genes of the $a c / s c$ complex encode basic helix-loop-helix proteins that heterodimerize with Daughterless (Da) to activate downstream genes required for neural fate. Transcription of $a c$ and $s c$ appears to be initiated by enhancers (Gomez-Skarmeta et al. 1995; Garcia-Garcia et al. 1999) and the expression is maintained throughout development by autoregulation mediated by $(\mathrm{Ac} / \mathrm{Sc})-\mathrm{Da}$ heterodimers binding to $\mathrm{E}$ boxes within the $a c / s c$ promoters (Martinez and Modolell 1991; Van Doren et al. 1991). Each enhancer interacts with specific transcription factors and promotes proneural expression in only one or a few proneural clusters. These factors, expressed in broader domains than the proneural clusters, define the bristle prepattern. Pannier (Pnr) belongs to the GATA-1 family of transcription factors (Ramain et al. 1993; Heitzler et al. 1996) and activates proneural expression required for development of the dorsocentral (DC) sensory bristles through binding to the DC enhancer (Garcia-Garcia et al. 1999) located at 4 and $30 \mathrm{~kb}$ from $a c$ and $s c$, respectively. Consequently, the loss of function pnr alleles fail to activate $a c / s c$ and lack DC sensory bristles. Chip is a ubiquitous nuclear protein required for maximal activation by diverse remote enhancers (Morcillo et al. 1996, 1997; Torigoi et al. 2000). It was shown that it physically interacts both with Pnr and the (Ac/Sc)-Da heterodimers and facilitates enhancer-promoter communication (Bulger and Groudine 1999; Dorsett 1999) during Pannier-driven neural development (Ramain et al. 2000).

In our current study, we present genetic interactions between osa, pnr, and Chip that reflect direct interactions between Osa and Pnr and between Osa and Chip. We show that Osa negatively regulates neural expression because loss of osa function exhibits increased expression of $a c / s c$ and an excess of DC sensory bristles. Pnr and Chip have been previously identified as essential proteins of a proneural complex that activates $a c / s c$ during neural development. Our study reveals that Pnr and Chip are also essential in recruiting Osa during neural repression. Hence, our study provides insights into how chromatin remodeling activity might be targeted to specific promoter sequences to regulate enhancer-promoter communication during development.

\section{Results and Discussion}

Chip $^{E}$ is a viable allele of Chip that is associated with a point mutation in the LIM-interacting domain (LID), which specifically reduces interaction with the bHLH proteins Ac, Sc, and Da. As a consequence, the Chip ${ }^{E}$ mutation disrupts the functioning of the proneural com- 
plex encompassing Chip, Pnr, Ac/Sc, and Da (Ramain et al. 2000). A homozygous Chip ${ }^{E}$ mutant shows thoracic cleft and loss of the DC bristles, similar to loss of function pnr alleles (Fig. 1B).

To identify new factors that regulate this proneural complex, we screened for second-site modifiers of the Chip ${ }^{E}$ phenotypes (I. Biryukova and P. Heitzler, unpubl.). We found one allele of osa $\left(o s a^{E 17}\right)$ among the putative mutants. Osa ${ }^{E 17}$ corresponds to a loss-of-function allele, and homozygous embryos die with normal cuticle patterning. Both $o s a^{E 17}$ and null alleles of osa losab15 or os $a^{14060}$ ) enhance the cleft but suppress the loss of DC bristle phenotypes of Chip ${ }^{E}$ flies. Indeed, Chip ${ }^{E}$ flies with only one copy of $\operatorname{Osa}^{+}\left(\mathrm{Chip}^{E} ; O s a^{616} /+\right)$ are weak and sterile but show wild-type DC bristle pattern (Fig. 1C).

These genetic interactions suggest that Osa can antagonize the function of Pnr. Moreover, overexpressed Osa (+/UAS-osa;Gal4-pnr ${ }^{M D 237} /+$ ) induces a thoracic cleft and the loss of DC bristles (Collins et al. 1999; our current study) similar to the loss-of-function pnr alleles (Heitzler et al. 1996; Garcia-Garcia et al. 1999). In contrast, loss-of-function osa alleles display an excess of DC bristles similar to overexpressed Pnr (Haenlin et al. 1997). For example, $\left(o s a^{14060} /+\right)$, $\left(o s a^{616} /+\right)$, and $\left(o s a^{E 17} /+\right)$ flies exhibit respectively $2.35 \pm 0.12,2.38 \pm 0.12$, and

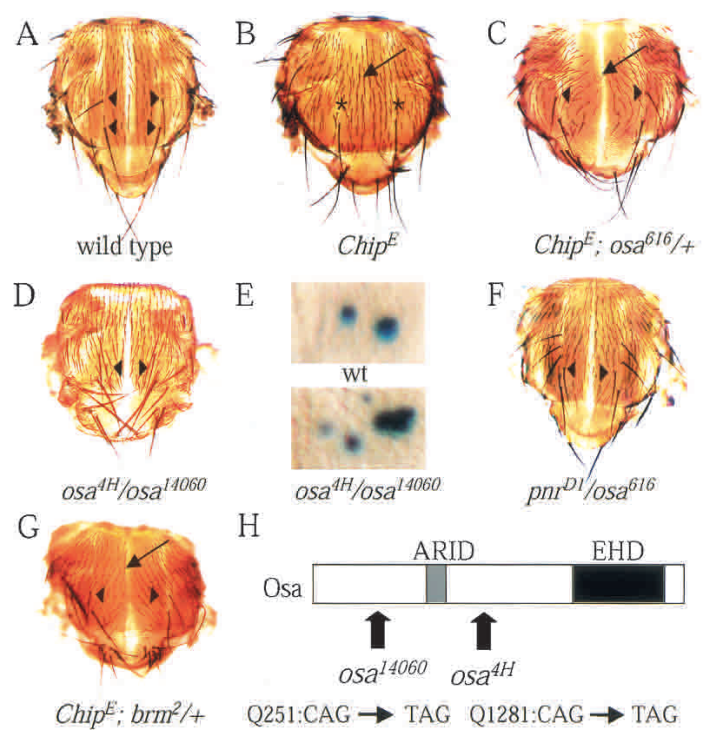

Figure 1. osa mutants genetically interact with pnr and Chip and display extra DC bristles. $(A-D, F, G)$ Dorsal thoraces of flies. $(A)$ Wild-type flies have four DC bristles (arrowhead). $(B) C_{\text {Chip }}^{E}$ flies resemble hypomorphic pnr mutants lacking DC bristles (asterisks) and bearing a thoracic cleft (arrow). (C) Null alleles of osa enhance the cleft (arrow) but suppress the lack of DC bristles (arrowhead) in Chip ${ }^{E}$ flies $\left(C_{\text {hip }}^{E} / C_{\text {Chip }}^{E} ;+/ o s a^{616}\right)$. $(D, E)$ Hypomorphic transallelic combination of osa (os $\left.a^{4 H} / o s a^{14060}\right)$ exhibits extra DC bristles $(D$, arrowhead) associated with extra DC precursors $(E)$ as revealed by A101 lacZ staining in comparison with staining of wild-type disc. $(F)$ os $a^{616}$ behaves as dominant enhancer of the excess of DC bristles associated with the $p n r^{D}$ encoding constitutive activators of $a c / s c$ $\left(p r^{D 1} / o s a^{616}\right)$. (G) Reducing the amount of brm also enhances the cleft (arrow) but suppresses the lack of DC bristles (arrowhead) in Chip ${ }^{E}$ flies (Chip ${ }^{E} /$ Chip $^{E}$; $+/$ brm $\left.^{2}\right)$. (H) Molecular characterization of the $0 s a^{14060}$ and $o s a^{4 H}$ alleles. osa encodes a protein carrying an ARID DNA binding domain (gray box) and an EHD (black box) conserved in human and Caenorhabditis elegans homologs. osa ${ }^{14060}$ and $O S a^{4 H}$ encode truncated Osa protein where the EHD is lacking. The glutamine mutated to a stop codon in $0 s a^{14060}\left(\mathrm{Q} \mathrm{n}^{\circ} 251\right)$ and ${ }^{o s} a^{4 H}\left(\mathrm{Q} \mathrm{n}^{\circ} 1281\right)$ is indicated by an arrow.
$2.43 \pm 0.17$ DC bristles per heminotum /Oregon wildtype flies have $2.00 \mathrm{DC}$ bristles/heminotum). Furthermore, transallelic combination of $0 s a^{14060}$ with the hypomorphic $O \mathrm{Sa}^{4 H}\left(\mathrm{osa} \mathrm{S}^{4 H} / \mathrm{osa}^{14060}\right)$ accentuates the excess of DC bristles (Fig. 1D) compared with $\left(\mathrm{osa}^{14060} /+\right)$. $\left(o s a^{4 H} / o s a^{14060}\right)$ flies display $4.17 \pm 0.19 \mathrm{DC}$ bristles per heminotum. On the other hand, $\left(\mathrm{osa}^{4 H} / \mathrm{osa}^{4 H}\right)$ flies display $2.50 \pm 0.11 \mathrm{DC}$ bristles per hemithorax. The development of the extra DC bristles revealed by phenotypic analysis was compared with the positions of the DC bristle precursors detected with a LacZ insert, A101, in the neuralized gene (Boulianne et al. 1991) that exhibits staining in all sensory organs (Huang et al. 1991). In $\left(\right.$ os $a^{1400} /$ os $\left.a^{4 H}\right)$ discs, additional DC precursors are observed that lead to the excess of DC bristles (Fig. 1E). The $p n r^{D}$ alleles encode Pnr proteins carrying a single amino acid substitution in the DNA binding domain that disrupts interaction with the U-shaped (Ush) antagonist (Cubadda et al. 1997; Haenlin et al. 1997). Consequently, $\mathrm{Pnr}^{\mathrm{D}}$ constitutively activates $a c / s c$, leading to an excess of DC bristles. We found that this excess is accentuated when osa function is simultaneously reduced $\left(p n r^{D 1}\right)$ osa ${ }^{616}$ in Fig. 1F).

As osa shows genetic interactions with trithorax group genes encoding components of the Brm complex like moira (mor) and brm (Collins et al. 1999; Crosby et al. 1999; Vazquez et al. 1999), we investigated whether mutations in mor and brm suppress the Chip ${ }^{E}$ phenotype. We found that loss of one copy of brm ${ }^{+}$in Chip $^{E}$; $\mathrm{brm}^{2} /+\mid$ flies suppresses the lack of DC bristles observed in Chip ${ }^{E}$ flies (Fig. 1G), similar to loss of one copy of osa $^{+}$ (Fig. 1C). This shows that brm and osa both act during Pnr-dependent patterning, in agreement with the fact that they have been shown to be associated in the Brm complex. In contrast, reducing the amount of Mor by half $\left[\left(\right.\right.$ Chip $^{E} ;$ mor $\left.^{1} /+\right)$ flies $]$ is not sufficient to modify the $C_{\text {Chip }}^{E}$ phenotype (data not shown). This does not definitely exclude the possibility that mor is directly or indirectly involved, via the Brm complex, in Pnr-dependent patterning.

The complete osa open reading frame of 2715 amino acids and the intronic splicing signals were PCR amplified from genomic DNA prepared from homozygous embryos $\left(o s a^{E 17}\right.$ and $\left.o s a^{14060}\right)$ and homozygous first instar larvae $\left(o s a^{4 H}\right)$. For $O s a^{14060}$ and $O s a^{4 H}$, the sequence analysis revealed a single mutation in the $\mathrm{N}$ terminus that causes a glutamine to stop codon substitution (Q $\mathrm{n}^{\circ} 251$ for os $^{14060}$; Q n ${ }^{\circ} 1281$ for os ${ }^{4 H}$; Fig. $\left.1 \mathrm{H}\right)$. The conceptual translation of $\mathrm{os}^{14060}$ leads to a truncated Osa protein lacking both functional domains, whereas $\mathrm{Osa}^{4 \mathrm{H}}$ retains the ARID domain but lacks the C-terminal EHD (Fig. 1H). Wild-type osa function is necessary for patterning of the DC bristles. Although $0 s a^{E 17}$ behaves as a stronger allele than $O s a^{14060}$ and $O s a^{4 H}$, we were unable to molecularly identify the mutation. Hence, the $O s a^{E 17}$ phenotype may result from a mutation in regulatory sequences that affects osa expression.

We have previously shown that a complex containing Pnr, Chip, and the (Ac/Sc)-Da heterodimer activates proneural expression in the DC proneural cluster and promotes development of the DC macrochaetae (Ramain et al. 2000). Osa and Pnr/Chip have antagonistic activities during development because loss of osa function $\left(o s a^{4 H}\right.$ and $o s a^{14060}$ ) displays additional DC bristles. However, as our current study reveals that osa genetically interacts with pnr and Chip, we asked whether Osa physically 
A
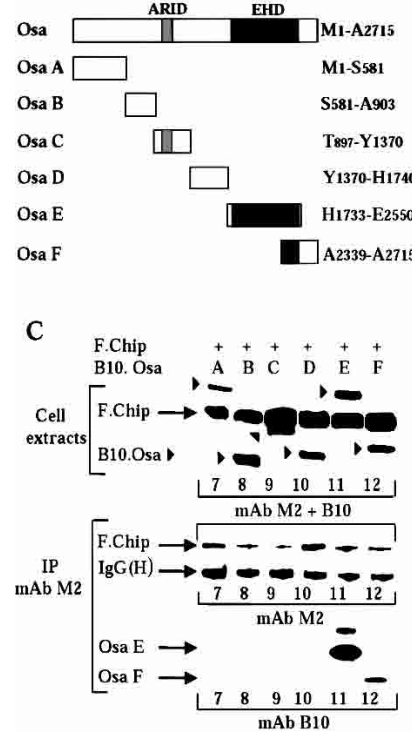

B

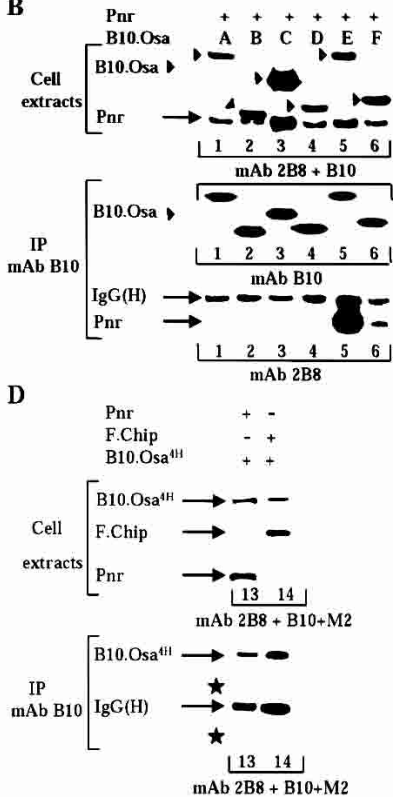

Figure 2. Osa physically interacts with Pnr and Chip through its C-terminal EHD. Osa ${ }^{4 \mathrm{H}}$ consequently no longer interacts with Pnr and Chip. (A) Schematic drawing of the Osa domains used throughout the current study. They are as follows: Osa A, Meth1/Ser581, Osa B, Ser581/Ala903; Osa C, Thr897/Tyr1370; Osa D, Tyr1370/ His1740; Osa E, His1733/Glu2550; Osa F, Ala2339/Ala2715. $(B, C)$ Osa interacts with Pnr $(B)$ and with Chip $(C)$. In each case, an immunoblot of a representative set of transfected cells extracts is shown in the upper part of the panels. Immunoprecipitations of transfected cell extracts are shown in the lower part of the panels. The transfected expression vectors are shown at the top of the panels. The B10 and M2 mouse antibodies used to immunoprecipitate the extracts are shown at the left of the panels and the antibodies used to reveal the blots are indicated at the bottom. Pnr is recognized by the $2 \mathrm{~B} 8$ monoclonal antibody, the B10-tagged Osa domains are detected with the B10 antibody, and the full-length flagged Chip is detected with the M2 antibody. The locations of the Osa domains are indicated by arrowheads. The locations of the flagged Chip and Pnr proteins as well as those of the immunoglobulin heavy chain $[\mathrm{IgG}(\mathrm{H})]$ are indicated at the sides. $(D) \mathrm{Osa}^{4 \mathrm{H}}$ no longer interacts with Pnr or Chip. The layout is as in $B$ and $C$. The stars highlight the absence of Pnr (lane 13) and F-Chip (lane 14) coimmunoprecipitating with immunoprecipitated B10.Osa ${ }^{4 \mathrm{H}}$.

interacts with the Pnr and Chip proteins. We performed immunoprecipitations of protein extracts made from Cos cells cotransfected with expression vectors for tagged Osa and either Pnr or tagged Chip (Fig. 2B,C). Because Osa is a large protein, we used several expression vectors encoding contiguous domains of Osa (Fig. 2A, domains A-F). Osa coimmunoprecipitates with Pnr and Chip (Fig. 2B,C) and can be detected on Western blots with appropriate antibodies. The interactions appear to require the overlapping domains Osa E (His1733/ Glu2550) and Osa F (Ala2339/Ala2715; Fig. 2B,C) corresponding to the EHD. Enhancer-promoter communication during proneural activation and development of the DC bristles requires regulatory sequences scattered over large distances and appears to be negatively regulated by interaction of Pnr and Chip with Osa through the EHD. Interestingly, the EHD is not conserved in yeast. In yeast, the UAS sequences are generally close to the promoter and there is no requirement for long-distance interactions. This observation could support the idea that

the EHD is essential for long-distance enhancer-promoter communication. Alternatively, yeast may just lack proteins like Chip or Pnr.

The DNA-binding domain and the C-terminal region are essential for the function of Pnr during development of the DC sensory organs (Ramain et al. 1993). The $p n r^{V X 1}$ and $p n r^{V X 4}$ alleles (collectively $p n r^{V X 1 / 4}$ ) are characterized by frameshift deletions that remove two C-terminal $\alpha$-helices and result in reduced proneural expression and loss of DC bristles.

We thus investigated the molecular interactions between Osa and Pnr ${ }^{D 1}$ and between Osa and Pnr ${ }^{\mathrm{VX} 1}$. We found that the $\mathrm{Pnr}^{\mathrm{D} 1}$ protein interacts with the EHD as efficiently as wild-type Pnr (Fig. 3A,B, lanes 1,2). In contrast, the physical interaction is disrupted when the $\mathrm{C}$ terminus of Pnr encompassing the $\alpha$-helices is removed (Fig. 3A,B, lanes 1,3). Because the $\mathrm{C}$ terminus of Pnr is required for the Pnr-Osa interaction in transfected cells extracts, we tested the abilities of in vitro translated 35Slabeled Osa domains to bind to GST-C ${ }^{\mathrm{T}} \mathrm{Pnr}$ attached to glutathione-bearing beads (Fig. 3D, lane 4). We found that only Osa $\mathrm{E}$ and Osa $\mathrm{F}$ interact with the $\mathrm{C}$ terminus of Pnr (Fig. 3D; data not shown). As expected, GST-C ${ }^{\mathrm{T}}$.

A Pnr
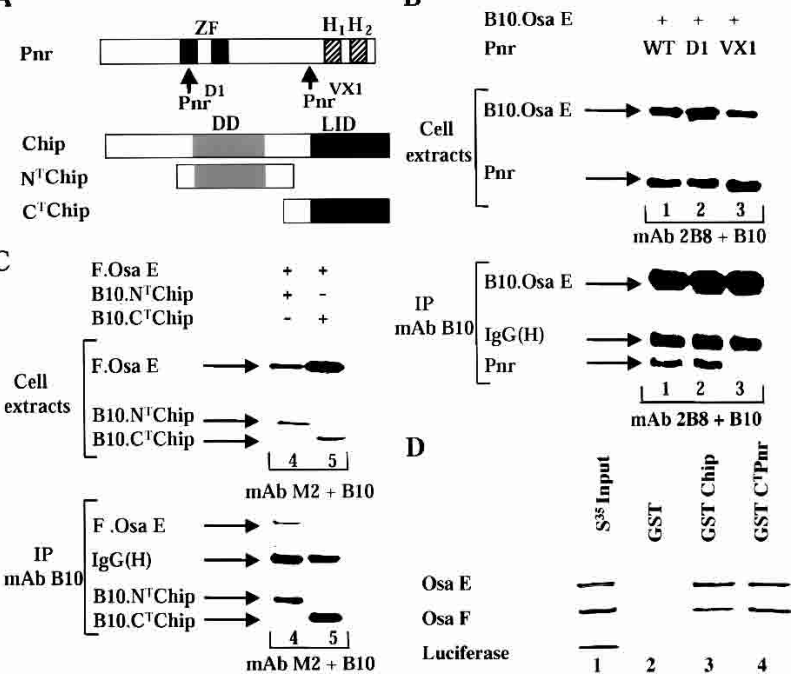

Figure 3. The C-terminal domain of Pnr encompassing the two helical structures and the $\mathrm{N}$-terminal homodimerization domain of Chip mediate interactions with Osa. (A) Structural features of the Pnr and Chip proteins used in the present study. The two zinc fingers (black boxes; ZF) and two amphipathic $\alpha$-helices (hatched boxes; H1 and H2) of Pnr are shown. The mutations associated with the proteins of the alleles $p n r^{D 1}$ and $p n r^{V X 1}$ are localized within the first zinc finger and in the $\mathrm{N}$ terminus of the helices, respectively. The N-terminal homodimerization domain of Chip $\left(\mathrm{N}^{\mathrm{T}}\right.$ Chip; gray box; DD) and the C-terminal LIM-interacting domain $\left(\mathrm{C}^{\mathrm{T}}\right.$ Chip; black box; LID) are also shown. $(B, C)$ The C-terminal domain of Pnr containing the $\alpha$ helices $(B)$ and the $\mathrm{N}$-terminal homodimerization domain of Chip $(C)$ mediate the interactions with Osa. Immunoprecipitations of transfected cell extracts are shown. The layout is as described in Figure 2B-D. The B10 Chip $\mathrm{C}^{\mathrm{T}}$ comigrates with the immunoglobulin light chain and was detected in $B$ (lane 5) using a peroxidase-conjugated protein A-Sepharose. $(D)$ Autoradiographs of SDS-PAGE gels from representative affinity chromatography experiments with the GST Chip beads (lane 3), the GST C ${ }^{\mathrm{T}} \mathrm{Pnr}$ (lane 4), or the GST control beads (lane 2) and in vitro translated ${ }^{35} \mathrm{~S}$ proteins are indicated on the left. One-tenth of the ${ }^{35} \mathrm{~S}$ input is shown in lane 1. Luciferase is used as a negative input. Experiments were performed three times and, with all proteins except luciferase, 50-fold more protein bound to GST C ${ }^{\mathrm{T}} \mathrm{Pnr}$ and GST Chip than to GST control. 
Heitzler et al.

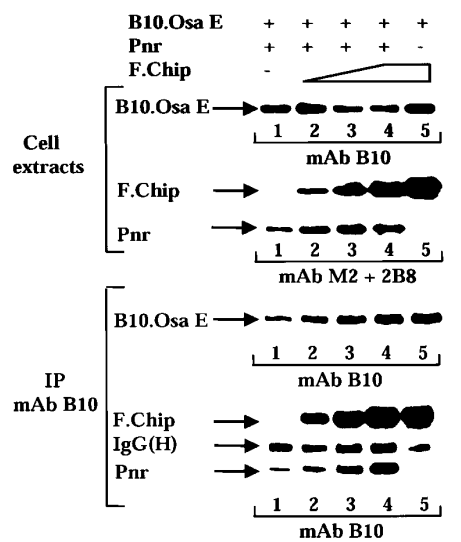

Figure 4. Chip and Osa do not compete for binding to Pnr. The immunoprecipitation of transfected cell extracts is shown. The layout is as described in Figures 2B-D and 3B-C. The top part of the figure shows the immunoblot of a representative set of transfected cell extracts where a constant amount of Pnr and a constant amount of the tagged Osa E have been expressed in the presence of increasing amounts of F.Chip. The bottom part displays the immunoprecipitation of the protein extracts with a constant amount of immunoprecipitated Osa $\mathrm{E}$ and an increasing amount of coimmunoprecipitating Pnr when the amount of coimmunoprecipitating F Chip is increasing.

Pnr did not bind the luciferase input and none of the ${ }^{35} \mathrm{~S}$ proteins bound GST control beads (Fig. 3D, lanes 4,2). We also further investigated the interaction between Chip and Osa and we found that Osa associates with the $\mathrm{N}$-terminal homodimerization domain of Chip (Fig. 3A,C, lane 4), also required for the interaction between Chip and Pnr. Furthermore, Osa E and Osa F bound also to immobilized GST-Chip (Fig. 3D, lane 3). We found that deletion of the $\alpha$ helix $\mathrm{Hl}$ disrupts the interactions between Pnr and Osa (data not shown). Interestingly, the same deletion also disrupts the interaction with Chip (Ramain et al. 2000). Therefore, the functional antagonism between Chip and Osa during neural development may result from a competition between these proteins for association with Pnr. Alternatively, the deletion of $\mathrm{H} 1$ may affect the overall structure of the $\mathrm{C}$ terminus of Pnr and disrupt the physical interactions with Chip and Osa. To discriminate between these hypotheses, we performed immunoprecipitations of protein extracts containing a constant amount of Pnr, a constant amount of the tagged Osa E domain, and increasing concentrations of Chip (Fig. 4). Pnr immunoprecipitates with immunoprecipitated tagged Osa E and the amount of Pnr immunoprecipitated increases in the presence of increasing concentrations of Chip. The presence of increasing amounts of Chip does not inhibit the Osa-Pnr interaction as would be expected if Osa and Chip were to compete for binding to Pnr. In contrast, it suggests that Chip and Pnr act together to recruit Osa and to target its activity and possibly the activity of the Brm complex to the $a c / s c$ promoter sequences.

Using expression vectors encoding contiguous domains of Osa, we showed that the EHD of Osa mediates interactions with Pnr and Chip. Because the EHD is lacking in the truncated $\mathrm{Osa}^{14060}$ and $\mathrm{Osa}^{4 \mathrm{H}}$, we hypothesized that the loss of interaction with Pnr and Chip are responsible for the excess of DC bristles observed in $o s a^{4 H}$ and $o s a^{14060}$. However, it is possible that we disrupted an additional $\mathrm{N}$-terminal interaction domain in constructing the expression vectors. To exclude this possibility, we performed immunoprecipitations of protein extracts made from Cos cells cotransfected with expression vectors for a tagged $\mathrm{Osa}^{4 \mathrm{H}}$ and either Pnr or a tagged Chip. We found that the truncated $\mathrm{Osa}^{4 \mathrm{H}}$ does not interact either with Pnr or with Chip, indicating that there is no dimerization domain in the $\mathrm{N}$ terminus of Osa (Fig. $2 \mathrm{D}$, lanes 13,14).

To investigate whether these interactions between Osa, Pnr, and Chip function in vivo during DC bristle development, we have examined the effects of both loss of function and overexpression of osa on the activity of a LacZ reporter whose expression is driven by a minimal promoter sequence of $a c$ fused to the DC enhancer (transgenic line DC:ac-LacZ; Fig. 5A; Garcia-Garcia et al. 1999; Ramain et al. 2000). We found that expression of the LacZ transgene is increased in $\mathrm{osa}^{14060} / \mathrm{osa}^{4 H}$ wing discs when compared with the wild-type control (Fig. 5A, a,b). For overexpression experiments, we used the UAS/GAL4 system (Brand and Perrimon 1993) using as a driver the $p n r^{M D 237}$ strain that carries a GAL4-containing transposon inserted in the pnr locus (driver: pnrGal4). This insert gives an expression pattern of Gal4 indistinguishable from that of pnr (Calleja et al. 1996; Heitzler et al. 1996). We found that overexpressed Osa leads to a strong reduction of $L a c Z$ staining in the DC

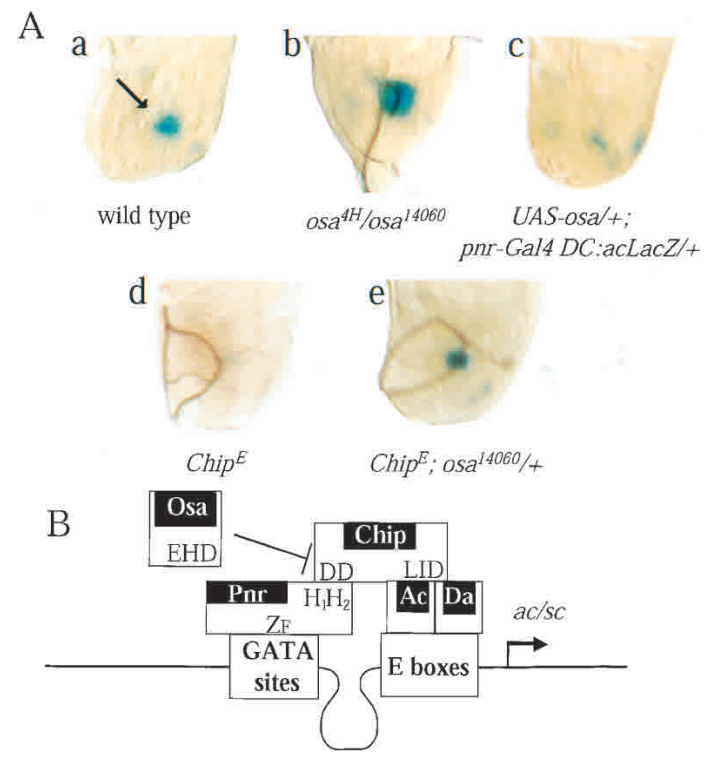

Figure 5. Osa regulates proneural expression through the DC-specific enhancer of the $a c / s c$ complex. Late-third instar thoracic discs show lacZ expression in the DC area (Gomez-Skarmeta et al. 1995). In each case, the reaction was left for $1 \mathrm{~h}$ at $22^{\circ} \mathrm{C}$. $(A$, ) Wild-type pattern $\left(w^{1118} /+\right.$; DC:ac-LacZ/+) with DC expression (arrow). (b) Hypomorphic transallelic combination of osa (DC:ac-LacZ osa ${ }^{4 H}$ ) ${ }^{O S a^{14060}}$ ) promotes overexpression of LacZ. (c) Overexpressed Osa mimics the effects of $\mathrm{Chip}^{E}\left[\mathrm{UAS}-\mathrm{Osa}{ }^{+} /+\right.$; +/DC:ac-LacZ pnr $^{M D 237}$ (Gal4)]. (d) In Chip ${ }^{E}$ flies (Chip ${ }^{E} /$ Chip $^{E}$; DC:ac-LacZ/+), the $l a c Z$ expression driven by the enhancer is strongly reduced. (e) Lowering the dosage of ${ } s a^{+}$suppresses the effects of Chip ${ }^{E}\left(\right.$ Chip $\left.^{E}\right)$ $C_{\text {Chip }}{ }^{*}$; DC:ac-LacZ/osa ${ }^{14060}$ ) and restores the activity of the promoter sequences (cf. $a, d)$. (B) A model on how the proneural complex encompassing Pnr, Chip, and the (Ac/Sc)-Da heterodimer is regulated by Osa. The proneural complex activates $a c / s c$ and is negatively regulated by interactions of Pnr and Chip with Osa. These interactions are mediated by the $\mathrm{C}$-terminal domain of Pnr containing two $\alpha$ helices $(\mathrm{H} 1 \mathrm{H} 2)$, the $\mathrm{N}$-terminal homodimerization domain (DD) of Chip, and the C-terminal EHD of Osa. 
area (Fig. 5A, a,c), consistent with the lack of DC bristles (Collins et al. 1999; data not shown). Thus, overexpressed Osa represses activity of the ac promoter sequences required for DC $a c / s c$ expression and development of the DC bristles. It has been previously reported that wingless expression is also required for patterning of the DC bristles (Phillips and Whittle 1993). However, the repressing effect of Osa on development of the DC bristles is unlikely to be the result of an effect of Osa on wingless expression because overexpressed Osa driven by $p n r^{M D 237}$ has no effect on the expression of a $L a c Z$ reporter inserted into the wingless locus (data not shown; Kassis et al. 1992; Collins and Treisman 2000). Thus, Osa acts through the DC enhancer of the $a c / s c$ promoter sequences to repress $a c / s c$ and neural development.

Chip ${ }^{E}$ disrupts the enhancer-promoter communication and strongly affects expression of the $L a c Z$ reporter driven by the ac promoter linked to the DC enhancer (Fig. 5A, d; Ramain et al. 2000). Because null alleles of osa suppress the loss of DC bristles displayed by Chip ${ }^{E}$, we investigated the consequences of reducing the dosage of osa in Chip ${ }^{E}$ flies. We found that the expression of the LacZ reporter is not affected in Chip ${ }^{E}$ flies when Osa concentration is simultaneously reduced $\left(\right.$ Chip $^{E} /$ Chip $^{E}$; DC:ac-LacZ/osa ${ }^{14060}$; Fig. 5A, e).

In conclusion, we have previously shown that Pnr function during proneural patterning is regulated by interaction with several transcription factors (Fig. 5B). Pnr function is negatively regulated by Ush that interacts with its DNA-binding domain (Haenlin et al. 1997). Chip associates with the $\mathrm{C}$ terminus of Pnr, bridging Pnr at the DC enhancer with the AC/Sc-Da heterodimers bound at the proneural promoters, thus activating proneural gene expression (Ramain et al. 2000). Our current study reveals that Pnr function can also be regulated by interaction with Osa. Thus, Osa activity is specifically targeted to $a c / s c$ promoter sequences and the binding of Osa therefore has a negative effect on Pnr function, leading to reduced expression of the proneural ac/sc genes. Osa belongs to Brm complexes, which are believed to play an essential role during chromatin remodeling necessary for gene expression. For example, in vitro transcription experiments with nucleosome assembled human $\beta$-globin promoters have shown that the BRG1 and BAF155 subunits of the mammalian SWI/SNF homolog are essential to target chromatin remodeling and promote transcription initiation mediated by GATA-1 (Kadam et al. 2000). In contrast to what was observed in vitro, our results suggest that in vivo the SWI/SNF complexes can also act to remodel chromatin in a way that represses transcription. Alternatively, the observed repression of proneural genes may simply define a novel function of Osa, independent of chromatin remodeling.

\section{Materials and methods}

All recombinant DNA work was performed according to standard procedures (Sambrook et al. 1989). Details concerning plasmid constructions are available on request.

Fly stocks and genetics

Alleles used were $o s a^{4 H}$ (Kaminker et al. 2001), osa ${ }^{616}$ (Treisman et al. 1997), osa ${ }^{14060}$ (gift of M. Hoch), mor $^{1}$, and brm $^{2}$ (FlyBase). The original $o s a^{4 H}$ chromosome leads to embryonic lethality. We found that this lethality is associated with a separable and distal mutation. Thirty hemithoraces were examined for each of the combinations presented in Figure
1, but for each genotype the phenotype was found to be remarkably similar from fly to fly.

PCR analysis of the osa mutants

Genomic DNA for PCR analysis was extracted from homozygous $O S a^{E 17}$, osa ${ }^{14060}$ embryos, and homozygous $o s a^{4 H}$ first instar larvae.

Plasmid constructions

The expression vectors pXJ $\mathrm{Pnr}^{+}$pXJ $\mathrm{Pnr}^{\mathrm{Dl}}$ and $\mathrm{pXJ} \mathrm{Pnr}^{\mathrm{VX} 1}$, encoding truncated versions of wild-type $\mathrm{Pnr}, \mathrm{Pnr}^{\mathrm{Dl}}$, and $\mathrm{Pnr}^{\mathrm{VX} 1}$, are described in Haenlin et al. (1997). The sequences encoding the domains of Osa (Osa A, Meth1/Ser581; Osa B, Ser581/Ala903; Osa C, Thr897/Tyr1370; Osa D, Tyr1370/His1740; Osa E, His1733/Glu2550; Osa F, Ala2339/Ala2715| and $\mathrm{Osa}^{4 \mathrm{H}}$ were amplified by PCR and inserted into the vectors pXJB, pXJF for transient transfections in Cos cells (Ramain et al. 2000). pXJB and pXJF encode fusion proteins carrying at their $\mathrm{N}$ terminus the B epitope of the estrogen receptor (pXJB: RPNSDNRRQGGRERL) and the Flag epitope (pXJF: DYKDDDDK), respectively. The vectors encoding the Flag-tagged Achaete, the B-tagged $\mathrm{N}$-terminal, and the B-tagged C-terminal domain of Chip are described in Ramain et al. (2000).

DNA transfections, immunoprecipitations, Western blot analysis, and GST pull-down assays

Cos cell transfections, protein extract preparations, immunoprecipitations, and Western blot analysis were performed as described in Haenlin et al. (1997). The protein extracts were immunoprecipitated with the B10 or M2 antibodies that recognize the B and Flag epitopes encoded by the pXJB and pXJF vectors. GST pull-down assays were performed as in Torigoi et al. (2000).

Staining for $\beta$-galactosidase activity

Wing discs were stained as described in Cubadda et al. (1997). A101 contains a LacZ gene insert at the neuralized locus (Boulianne et al. 1991) and exhibits staining in all sensory organ precursors (Huang et al. 1991). The transgenic strain DC:ac-LacZ is described in Ramain et al. (2000).

\section{Acknowledgments}

We thank Irwin Davidson and Luc Maroteaux for critical reading of the manuscript; Michael Hoch, Jim Kennisson, Tim Lebestky, Juan Modolell, Jessica Treisman, and the Bloomington stock center for reagents; Claudine Ackermann, Nadine Arbogast, and Marie-Louise Nullans for excellent technical assistance; and the sequencing, oligonucleotide synthesis, and cell culture services of the IGBMC. Inna Biryukova is supported by a fellowship from the Fondation pour la Recherche Médicale (FRM). This work was supported by grants from the CNRS, INSERM, the Hôpital Universitaire de Strasbourg, the Université Louis Pasteur, the Association pour la Recherche contre le Cancer (ARC), and the Ministère de la Recherche et de la Technologie.

The publication costs of this article were defrayed in part by payment of page charges. This article must therefore be hereby marked "advertisement" in accordance with 18 USC section 1734 solely to indicate this fact.

\section{References}

Boulianne, G.L., de la Concha, A., Campos-Ortega, J., Jan, L.Y., and Jan, Y.N. 1991. The Drosophila neurogenic gene neuralised encodes a novel protein and is expressed in precursors of larval and adult neurons. EMBO J. 10: 2975-2983.

Brand, A.H. and Perrimon, N. 1993. Targeted gene expression as a means of altering cell fates and generating dominant phenotypes. Development 118: 401-415.

Bulger, M. and Groudine, M. 1999. Looping versus linking: Toward a model for long distance gene activation. Genes \& Dev. 13: 2465 2477.

Calleja, M., Moreno, E., Pelaz, S., and Morata, G. 1996. Visualization of gene expression in living adult Drosophila. Science 274: 252-255.

Collins, R.T. and Treisman, J.E. 2000. Osa-containing Brahma chromatin remodeling complexes are required for the repression of Wingless target genes. Genes \& Dev. 14: 3140-3152.

Collins, R.T., Furukawa, T., Tanese, N., and Treisman, J.E. 1999. Osa 


\section{Heitzler et al.}

associates with the Brahma chromatin remodeling complex and promotes the activation of some target genes. EMBO J. 18: 7029-7040.

Crosby, M.A., Miller, C., Alon, T., Watson, K.L., Verrijzer, C.P., Goldman-Levi, R., and Zak, N.B. 1999. The trithorax group gene moira encodes a Brahma-associated putative chromatin-remodeling factor in Drosophila melanogaster. Mol. Cell. Biol. 19: 1159-1170.

Cubadda, Y., Heitzler, P., Ray, R., Bourouis, M., Ramain, P., Gelbart, W., Simpson, P., and Haenlin, M. 1997. u-shaped encodes a zinc finger protein that regulates the proneural genes achaete and scute during formation of bristles in Drosophila. Genes \& Dev. 11: 3083-3095.

Dorsett, D. 1999. Distant liaisons: Long-range enhancer-promoter interactions in Drosophila. Curr. Opin. Genet. Dev. 9: 505-514.

Garcia-Garcia, M.J., Ramain, P., Simpson, P., and Modolell, J. 1999. Different contributions of pannier and wingless to the patterning of the dorsal mesothorax of Drosophila. Development 126: 3523-3532.

Gomez-Skarmeta, J.L., Rodriguez, I., Martinez, C., Culi, J., Ferres-Marco, D., Beamonte, D., and Modolell, J. 1995. Cis-regulation of achaete and scute shared enhancer-like elements drive their coexpression in proneural clusters of the imaginal discs. Genes \& Dev. 9: 1869-1882.

Haenlin, M., Cubadda, Y., Blondeau, F., Heitzler, P., Lutz, Y., Simpson, P., and Ramain, P. 1997. Transcriptional activity of Pannier is regulated negatively by heterodimerization of the GATA-DNA binding domain with a cofactor encoded by the u-shaped gene of Drosophila. Genes \& Dev. 11: 3096-3108.

Heitzler, P., Haenlin, M., Ramain, P., Calleja, M., and Simpson, P. 1996. A genetic analysis of pannier, a gene necessary for viability of dorsal tissues and bristle positioning in Drosophila. Genetics 143: 12711287

Huang, F., Dambly-Chaudière, C., and Ghysen, A. 1991. The emergence of sense organs in the wing disc of Drosophila. Development 111: 1087-1095.

Kadam, S., McAlpine, G.S., Phelan, M.L., Kingston, R.E., Jones, K.A., and Emerson, B.M. 2000. Functional selectivity of recombinant mammalian SWI/SNF subunits. Genes \& Dev. 14: 2441-2451.

Kaminker, J.S., Singh, R., Lebestky, T., Yan, H., and Banerjee, U. 2001. Redundant function of Runt domain binding partners, big brother and brother during Drosophila development. Development 128: 26392648.

Kassis, J.A., Noll, E., VanSickle, E.P., Odenwald, W.F., and Perrimon, N. 1992. Altering the insertional specificity of a Drosophila transposable element. Proc. Natl. Acad. Sci. 89: 1919-1923.

Kennison, J.A. and Tamkun, J.W. 1988. Dosage-dependent modifiers of Polycomb and Antennapedia mutations in Drosophila. Proc. Nat1. Acad. Sci. 85: 8136-8140.

Martinez, C. and Modolell, J. 1991. Cross-regulatory interactions between the proneural achaete and scute genes of Drosophila. Science 251: 1485-1487.

Modolell, J. 1997. Patterning of the adult peripheral nervous system of Drosophila. Perspect. Dev. Neurobiol. 4: 285-296.

Morcillo, P., Rosen, C., and Dorsett, D. 1996. Genes regulating the remote wing margin enhancer in the Drosophila cut locus. Genetics 144: 1143-1154.

Morcillo, P., Rosen, C., Baylies, M.K., and Dorsett, D. 1997. Chip, a widely expressed chromosomal protein required for segmentation and activity of a remote wing margin enhancer in Drosophila. Genes \& Dev. 11: 2729-2740.

Phillips, R.G. and Whittle, J.R.S. 1993. wingless expression mediates determination of peripheral nervous system elements in late stages of Drosophila wing disc development. Development 118: 427-438.

Pirrotta, V. 1997. Chromatin-silencing mechanisms in Drosophila maintain patterns of gene expression. Trends Genet. 13: 314-319.

Ramain, P., Heitzler, P., Haenlin, M., and Simpson, P. 1993. pannier, a negative regulator of achaete and scute in Drosophila, encodes a zinc finger protein with homology to the vertebrate transcription factor GATA-1. Development 119: 1277-1291.

Ramain, P., Khechumian, R., Khechumian, K., Arbogast, N., Ackermann, C., and Heitzler, P. 2000. Interactions between Chip and the Achaete/ Scute-Daughterless heterodimers are required for Pannier-driven proneural patterning. Mol. Cell 6: 781-790.

Sambrook, J., Fritsch, E.F., and Maniatis, T. 1989. Molecular cloning: A laboratory manual, 2nd ed. Cold Spring Harbor Laboratory Press, Cold Spring Harbor, NY.

Torigoi, E., Bennani-Baïti, I.M., Rosen, C., Gonzales, K., Morcillo, P.,
Ptashne, M., and Dorsett, D. 2000. Chip interacts with diverse homeodomain proteins and potentiates Bicoid activity in vivo. Proc. Nat1. Acad. Sci.. 97: 2686-2691.

Treisman, J., Luk, A., Rubin, G.M., and Heberlein, U. 1997. eyelid antagonizes wingless signaling during Drosophila development and has homology to the Bright family of DNA-binding proteins. Genes \& Dev. 11: 1949-1962.

Van Doren, M., Powell, P.A., Pasternak, D., Singson, A., and Posakony, J.W. 1991. The Drosophila extramacrochaetae protein antagonizes sequence-specific DNA-binding by daughterless/achaete-scute protein complexes. Development 113: 245-255.

Vazquez M., Moore L., and Kennison, J.A. 1999. The trithorax group gene osa encodes an ARID-domain protein that genetically interacts with the Brahma chromatin-remodeling factor to regulate transcription. Development 126: 733-742. 


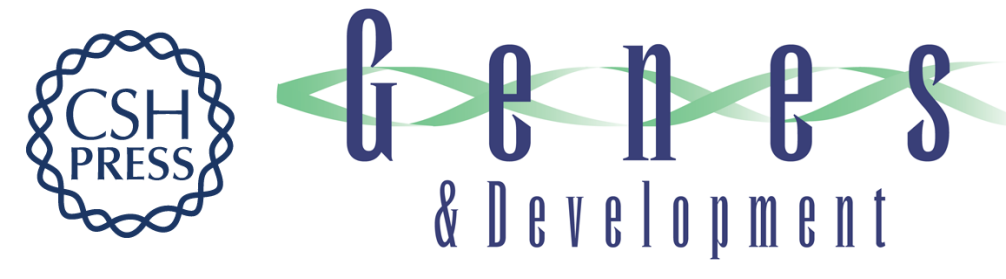

\section{Enhancer-promoter communication mediated by Chip during Pannier-driven proneural patterning is regulated by Osa}

Pascal Heitzler, Luc Vanolst, Inna Biryukova, et al.

Genes Dev. 2003, 17:

Access the most recent version at doi:10.1101/gad.255703

References This article cites 29 articles, 24 of which can be accessed free at: http://genesdev.cshlp.org/content/17/5/591.full.html\#ref-list-1

License

Email Alerting

Receive free email alerts when new articles cite this article - sign up in the box at the top Service right corner of the article or click here.

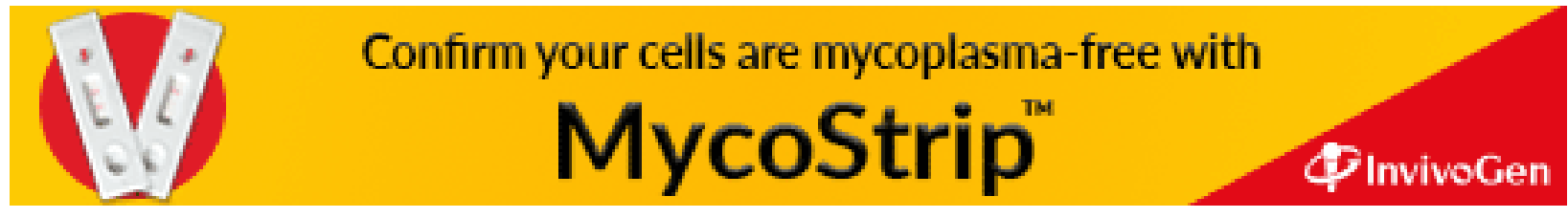

